\title{
OFDM Channel Estimation via Phase Retrieval
}

\author{
Philipp Walk \\ Department of Electrical Engineering \\ California Institute of Technology \\ MC 136-93, Pasadena, CA 91125 \\ Email:pwalk@caltech.edu
}

\author{
Henning Becker \\ Theoretical Information Technology \\ Technical University Munich \\ Theresienstrasse 90, 80290 München \\ Email: henning.becker@tum.de
}

\author{
Peter Jung \\ Communications \& Information Theory \\ Technical University Berlin (TUB) \\ Einsteinufer 25, 10587 Berlin \\ Email: peter.jung@tu-berlin.de
}

\begin{abstract}
Pilot-aided channel estimation is nowadays a standard component in each wireless receiver enabling coherent transmission of complex-valued constellations, only affected by noise and interference. Whenever these disturbances are sufficiently small and long data frames are used, high data rates can be achieved and the resource overhead due to the pilots vanishes asymptotically. On the other, it is expected that for the next generation of mobile networks not only data rate is in the main focus but also low latency, short and sporadic messages, massive connectivity, distributed\&adhoc processing and robustness with respect to asynchronism. Therefore a review of several wellestablished principles in communication has been started already.

A particular implication when using complex-valued pilots is that these values have to be known at the receiver and therefore these resources can not be used simultaneously for user data. For an OFDM-like multicarrier scheme this means that pilot tones (usually placed equidistantly according to the Nyquist theorem) are allocated with globally known amplitudes and phases to reconstruct the channel impulse response. Phases are designed and allocated globally which is in contrast to a distributed infrastructure.

In this work we present therefore a new phaseless pilot scheme where only pilot amplitudes need to be known at the receiver, i.e., phases are available again and can be used for various other purposes. The idea is based on a phase retrieval result for symmetrized and zero-padded magnitude Fourier measurements obtained by two of the authors. The phases on the pilot tones can now be used to carry additional user-specific data or compensate for other signal characteristics, like the PAPR.
\end{abstract}

\section{INTRODUCTION}

OFDM is a well-established common multicarrier modulation scheme for high-rate data transmission in time-invariant channels. It is used for example in IEEE-802.11a/g, LTE and DSL. New pulse-shaped multicarrier system which almost diagonalize typical mobile channels and are robust in the presence of asynchronism are proposed as candidates for the next generation of mobile systems. Most of these schemes use time-frequency multiplexing and are in this direction similar to OFDM. To obtain information about the channel for each subchannel pilot-based methods are used in most cases. Hence some resources are spent solely for channel estimation and this decreases the overall spectral efficiency. Obviously, whenever noise and interference contributions are sufficiently small, the channel does not change too rapidly, latency and energy is not an issue, high data rates can be achieved in this way. Asymptotically this overhead can then be neglected. On the other hand, the next generation of wireless mobile communication networks are much more than providing high data rates. It is expected that with the next cycles completely different communication scenarios will be in the focus where low latency, sporadic and short message support, asynchronous and non-orthogonal operation modes and distributed/adhoc and local processing are the major challenges.

Under these prerequisites it is important again to review several well-established principles of communication including also the tradeoff between pilot-overhead and amount of original information transfer. To come up with efficient blind estimation techniques which are able to operate on a short data frame basis is challenging. Intuitively it is clear that further apriori assumptions on the channel characteristics, like certain compressibility properties of the channel, for example sparsity, have to be exploited. From practical side, it is also difficult to implement such approaches efficiently in near future. However, a first step hereby would be the use of pilot phases for different tasks, i.e., to estimate the channel coefficients only by the magnitudes on the received pilot tones. The pilot phases are the available again for carring user-specific data. There are endless potential applications: (i) use pilot phases as a second data layer; (ii) use phase-shift keying alphabets (with constant and known amplitude) without specific pilot tones; (iii) use pilot phases for data-dependent signal compensation (like tonereservation for reducing the peak-to-average power ratio) and (iv) phase-preprocessing to avoid data-pilot interference in Offset-QAM-based multicarrier modulation. In addition, a central pilot-phase management is completely avoided, which has advantages in dense network structures with massive access. On the other hand, these benefits come not for free. Since conventional pilot-aided channel estimation is essentially the inversion of a linear problem it is in most cases stable, either by considering pilot placement under the Nyquist criterion or exploiting Compresses Sensing for recovery under sparsity priors. Phaseless recovery in turn is a quadratic problem and achieving stability in this particular setting is much more challenging.

First work in this direction has already be shown by the authors in [1]. The intention of this contribution is (i) to present our ideas in a more clear fashion; (ii) to further explain our scheme in the setting of phase retrieval and (iii) to discuss also differences to phase retrieval and finally to show some performance results achieved by an iterative estimation algorithm. The structure of the paper is as follows: First we give background information for the phase retrieval problem 
with further details for Fourier measurements. We explain some differences to our setup of symmetrized Fourier measurements. Using these investigations we propose a particular frame structure to support phaseless pilots for a semi-blind channel estimation (using only magnitudes). We will establish an iterative two-stage algorithm which can recover the channel impulse response up to global sign from magnitudes on the pilot tones. We will show simulations demonstrating that performance in terms of error rates.

Notation: We denote by capital letters integers and write for the set of the first $N$ integers $[N]=\{0,1, \ldots, N-1\}$. Bold letters denote vectors and bold capital letters refer to matrices. We denote by $\mathbf{F}_{N}$ the unitary $N$-dimensional discrete Fourier transform (DFT) giving element-wise for $k, l \in[N]$ as $\left(\mathbf{F}_{N}\right)_{l k}=\omega_{N}^{l k} / \sqrt{N}$ where $\omega_{N}=e^{-i 2 \pi / N}$ is the $N$ th root of unity. We will abbreviate $\mathbf{F x}=\hat{\mathbf{x}}=\mathbf{F}_{N} \mathbf{x}$ whenever the dimension of $\mathrm{x} \in \mathbb{C}^{N}$ is clear from context. The pointwise product is denoted by $\odot$ and the linear convolution by * where $\otimes$ refers to the circular convolution. We use further $\overline{\mathbf{x}}$ to denote complex conjugation of all vector coefficients and $\mathbf{R x}=\mathbf{x}_{-}=\left(x_{N-1}, \ldots, x_{0}\right)^{T}$ for the time reversal of $\mathbf{x} \in \mathbb{C}^{N}$. The $N \times N$ circular (down) shift matrix and time-reversal matrix is given by

$$
\mathbf{S}=\mathbf{S}_{N}=\left(\begin{array}{cccc}
0 & \cdots & 0 & 1 \\
1 & \cdots & 0 & 0 \\
\vdots & \ddots & & \vdots \\
0 & \cdots & 1 & 0
\end{array}\right) \quad, \quad \mathbf{R}=\mathbf{R}_{N}=\left(\begin{array}{cccc}
0 & \cdots & 0 & 1 \\
0 & \cdots & 1 & 0 \\
\vdots & \cdots & & \vdots \\
1 & \cdots & 0 & 0
\end{array}\right)
$$

We set $\mathbf{e}_{0}=(1,0, \ldots, 0)^{T}$ such that the circular shifts $\mathbf{S e}_{0}=$ $\mathbf{e}_{k}$ define the Euclidean basis in $N$-dimension $\left\{\mathbf{e}_{k}\right\}_{k=0}^{N-1}$.

\section{BACKGROUND}

\section{A. Background on Phase Retrieval}

The recovery of a signal from magnitude measurements is known as the phase retrieval problem. It has a long history beginning with the work of Gerchberg and Saxton in the 70's [2]. Later, Fienup [3] also considered this problem and gave explicit reconstruction algorithms for the phase from magnitude of Fourier measurements. One of the challenging tasks in phase retrieval is to determine the necessary and sufficient number of measurements for ensuring injectivity or even stability. Since one cannot distinguish between numbers of unit modulus from the magnitude of a linear measurement, such statements can hold here only up to a global phase.

a) Generic Measurements: Candes et.al. [4] have shown stable recovery w.h.p. of $N$-dimensional complex-valued signals from the magnitude of $\mathcal{O}(N \log N)$ Gaussian measurements. A more principal result from Balan et al. in [5] shows that injectivity holds for $4 N-2$ generic measurements. Moreover, they could give a fast reconstruction algorithm in [6]. Using projection methods, Mondragon and Voroninski could even show in [7] injectivity from $4 N-3$ generic measurements. However, a practical construction and implementation of measurements at this limiting number seems to be rather hard, but it serves as an ultimate theoretical bound. b) Fourier Measurements: It is well-known that for Fourier measurements further ambiguities, like for example conjugation, translation and reflection, can not be avoided. Further non-trivial ambiguities are characterized in [8]. Therefore, several modifications of "pure" Fourier measurements are proposed, depending on the application. For example, non-linear or interference-based approaches are considered to provide unique phase reconstruction. Wang [9] presented a method where interference with a known signal $\mathbf{y} \in \mathbb{C}^{N}$ helps to recover a signal $\mathbf{x} \in \mathbb{C}^{N}$ up to a global sign from only $3 N$ Fourier measurements $|\mathbf{F}(\mathbf{x}+\omega \mathbf{y})|^{2}$ where $\omega \in \mathbb{C}$ is a root of unity. To mitigate the ambiguities in phase retrieval the early approaches also try to explore sparsity properties on the signal. But it shows up that either oversampling nor sparsity helps to dissolve all ambiguities in the one-dimensional phase retrieval. $\mathrm{Lu}$ and Vetterli also use sparsity for spectral factorization of real valued impulse responses [10]. Moreover, they also give a reconstruction algorithm.

c) Sparse Phase Retrieval: With the advent of compressed sensing, generalized (generic) phase retrieval with sparsity priors came back in the research focus. For real $S_{-}$ sparse signals, Eldar and Mendelson [11] established stable recovery w.h.p. from $\mathcal{O}(S \log (e N / S))$ subgaussian measurements with high probability. The result [12] of Ehler, Fornasier and Sigl extends this to the complex case and they provide an explicit reconstruction algorithm, but the signal needs to have strong decay properties. Wang and $\mathrm{Xu}[13]$ stated injectivity for $S$-sparse complex-valued signals from $4 S-2$ generic measurements as long as $S<N$.

\section{B. Fourier Phase Retrieval and Conjugate-Symmetry}

To dissolve the inherent ambiguities in the phase retrieval problem with Fourier measurements, as (circular) timereversal, time-shift, and a global phase factor, see e.g. [8] and [14], it is also possible to consider or even construct (depending on the application) a further structure on the signal itself, which excludes the ambiguities right away, up to global sign. This structure is indeed known. Whenever the signal is invariant under circular time-reversal and complex conjugation, i.e. (see notations above),

$$
\mathbf{x}=\mathbf{S}(\overline{\mathbf{x}})_{-},
$$

then one calls $\mathbf{x}$ conjugate-symmetric and its Fourier transform is real, i.e., we have $\mathbf{F}_{N} \mathbf{x} \in \mathbb{R}^{N}$. In [15, Thm.2] we have shown that every signal $\mathbf{h} \in \mathbb{C}^{L}$ can be conjugate-symmetrized in $N=2 L+1+K$ dimensions by

$$
\mathcal{S}_{K}^{\circ}(\mathbf{h})=\left(\begin{array}{c}
0 \\
\mathbf{h} \\
\mathbf{0}_{K} \\
\overline{\mathbf{h}}_{-}
\end{array}\right)
$$

for any $K \in \mathbb{N}$. Due to the leading zero, we will in this paper denote the symmetrization operation by $\mathcal{S}_{K}^{\circ}$. Moreover, the chain "symmetrization, Fourier transform and absolutesquare", i.e, the map $\left|\mathbf{F} \mathcal{S}_{K}^{\circ}\right|^{2}: \mathbb{C}^{L} \rightarrow \mathbb{R}^{N}$ corresponds to the Fourier transform of the circular auto-convolution of $\mathbf{x}=$ $\mathcal{S}_{K}^{\circ}(\mathbf{h})$ for which it holds

$$
\mathbf{x}_{1} \circledast \mathbf{x}_{1}-\mathbf{x}_{2} \circledast \mathbf{x}_{2}=\left(\mathbf{x}_{1}-\mathbf{x}_{2}\right) \circledast\left(\mathbf{x}_{1}+\mathbf{x}_{2}\right)
$$


Note, this only holds for the auto-convolution and not for the auto-correlation. In fact, the auto-convolution equals the autocorrelation if and only if the conjugate-symmetry property (2) holds.

d) Stability Issues for Auto-Convolutions: The inverse problem of determining a function on the interval from its auto-convolution is also known as the "auto-convolution problem" and has been investigated in the literature rarely. Its ill-posedness has been analyzed for example in [16]. Using the auto-convolution on conjugate-symmetric signals such that $K \geq 2 L+1$, then the $K$ additional zeroes in (3) allow us to write the circular auto-convolution as a linear auto-convolution for which we could show in [17] and [18] a stability result, where the stability constant $\alpha=\alpha(2 S)$ depends only on the support length of the signal:

$$
\alpha\left\|\mathbf{h}_{1}-\mathbf{h}_{2}\right\|_{2}\left\|\mathbf{h}_{2}+\mathbf{h}_{1}\right\|_{2} \leq\left\|\left|\mathbf{F} \mathcal{S}_{K}^{\circ}\left(\mathbf{h}_{1}\right)\right|^{2}-\left|\mathbf{F} \mathcal{S}_{K}^{\circ}\left(\mathbf{h}_{2}\right)\right|^{2}\right\|_{2}
$$

for $\mathbf{h}_{1}, \mathbf{h}_{2} \in \Sigma_{S}^{L}\left(S\right.$-sparse vectors in $\left.\mathbb{C}^{L}\right)$. Although the conjugate-symmetric property seems to be a difficult constraint in certain applications, it can be very easily constructed in a wireless communication scenario as we will demonstrate below.

\section{Semi-Blind Channel Estimation}

In an OFDM-like system the user data payload is encoded, modulated and framed as sequences of complex data symbols $\hat{\mathbf{s}} \in \mathbb{C}^{N}$ and then transmitted by performing multiplexing in frequency (and time). Although our approach can even be formulated in a quite general context of (pulse-shaped) multicarrier schemes, we will base our exposition here on the simple setup of OFDM using a cyclic prefix with $N$ tones (subcarriers). We assume (and in praxis this is always the case) that $N$ is an even number, given by powers of 2 . Transforming the OFDM symbol back to time (recall that we use $\hat{\bullet}$ to denote Fourier transforms) and adding a cyclic prefix $C P$ of length $L$ to $\mathrm{s}$ one obtains at the receiver the noisy signal

$$
\mathbf{r}_{N+2 L-1}=\mathbf{h} *\left(\begin{array}{c}
C P \\
\mathbf{s}
\end{array}\right)+\mathbf{n}_{N+2 L-1}
$$

where $\mathbf{h} \in \mathbb{C}^{L}$ is the channel's impulse response of length $L$ and $\mathbf{n}_{N+2 L-1} \in \mathbb{C}^{N+2 L-1}$ denotes additive noise. The samples for $k \in\{L, \ldots, L+N\}$ in 6 represent then the circular convolution as:

$$
\mathbf{r}=\left(\begin{array}{c}
\mathbf{h} \\
\mathbf{o}_{N-L}
\end{array}\right) \circledast \mathbf{s}+\mathbf{n}
$$

\section{A. Conventional Pilot-aided Channel Estimation}

For the demodulation of the received symbols the channel coefficients have to be estimated. A well-investigated subject here is pilot-aided channel estimation, where the carrier positions $\mathcal{P}=\{D k \mid k \in[P]\}$ with $P D=N$ are used for pilot symbols $\hat{\mathbf{u}} \in \mathbb{C}^{P}$. It is clear that this corresponds to an ideal setup, since for real applications oversampling is used and only tones in the middle half of the spectrum can be allocated. Several approaches are known in literature to cope with this issue. To hold our exposition of the idea simple, we will ignore this here. On the remaining subcarriers $\mathcal{D} \subseteq[N] \backslash \mathcal{P}$ the userdata payload is modulated. Writing (7) in the Fourier domain gives therefore on the pilot tones

$$
\begin{aligned}
\hat{\mathbf{r}}_{\mathcal{P}} & =\sqrt{N} \operatorname{diag}(\hat{\mathbf{u}})\left(\mathbf{F}_{N}\left(\begin{array}{c}
\mathbf{h} \\
\mathbf{o}_{N-L}
\end{array}\right)\right)_{\mathcal{P}}+\hat{\mathbf{n}}_{\mathcal{P}} \\
& =\sqrt{P} \operatorname{diag}(\mathbf{u}) \mathbf{F}_{P}\left(\underset{\mathbf{o}_{P-L} \mathbf{h}}{\mathbf{h}}\right)+\hat{\mathbf{n}}_{\mathcal{P}}=: \mathbf{A}^{(\mathrm{c})} \mathbf{h}+\hat{\mathbf{n}}_{\mathcal{P}}
\end{aligned}
$$

since the Fourier transform of the transmit signal $\hat{\mathbf{s}}$ is constructed such that $\hat{\mathbf{s}}_{\mathcal{P}}=\hat{\mathbf{u}}$. Thus, the received pilots tones contain a linear measurement of the channel impulse response and the measurement matrix is given by $\mathbf{A}^{(\mathrm{c})}$. Here we assume $P \geq 2 L$ such that we can down-sample the Fourier transform $\mathbf{F}_{P}$ in $P$ dimensions.

e) Least-Squares Estimation:: The standard (Tikhonov regularized) least-squares estimates (LS) for $\mathbf{h} \in \mathbb{C}^{L}$ given the received pilots values $\hat{\mathbf{r}}_{\mathcal{P}}$ is:

$$
\left(\mathbf{A}^{(\mathrm{c})^{*}} \mathbf{A}^{(\mathrm{c})}+\tau\right)^{-1} \mathbf{A}^{(\mathrm{c}){ }^{*}} \hat{\mathbf{r}}_{\mathcal{P}}
$$

and is suitable as long as $\mathbf{A}^{(\mathrm{c})^{*}} \mathbf{A}^{(\mathrm{c})}$ is well-conditioned which depends on $P$ and $L$. The parameter $\tau$ will be chosen depending on the noise power $\sigma^{2}$, in particular for $\tau=\sigma^{2}$ this is also known as the Gauss-Markov estimator.

f) Estimation with Sparsity Priors: If we additionally know the length of the support $|\operatorname{supp}(\mathbf{h})| \leq S \ll L$, i.e., $\mathbf{h} \in \Sigma_{S}^{L}$ is $S$-sparse in $L$-dimensions, then we can even use "Compressed Sensing" methods to reconstruct $\mathbf{h}$, for example, by solving the convex basis pursuit denoising (BPDN) problem

$$
\min _{\mathbf{h} \in \mathbb{C}^{L}}\|\mathbf{h}\|_{1} \quad \text { s.t. } \quad\left\|\mathbf{A}^{(\mathrm{c})} \mathbf{h}-\hat{\mathbf{r}}_{\mathcal{P}}\right\|_{2} \leq \epsilon
$$

which yields a stable and robust solution if $\mathbf{A}^{(\mathrm{c})} \in \mathbb{C}^{N \times L}$ fulfils the RIP condition of order $2 k$ with sufficiently small RIP-constant (can be achieved w.h.p. by randomizing the pilot positions), see [19] and [20]. Here $\epsilon^{2}$ is usually proportional to the noise power $\sigma^{2}$ and has to be tuned depending on several system parameters. In real-world wireless applications this problem has to be solved by efficient greedy methods.

\section{B. Phaseless Channel Estimation}

In our proposed scheme now, we consider two OFDM symbols $\hat{\mathbf{s}}_{1}$ and $\hat{\mathbf{s}}_{2}$ carrying the same pilot tones $\hat{\mathbf{u}}$ and being transmitted over the same channel $\mathbf{h} \in \mathbb{C}^{L}$. In the shortest setup both symbols are sent consecutively. The key feature of our scheme is to use in the second symbol the same pilots $\hat{\mathbf{u}}$ and send them time reversed and complex conjugated $\left(\overline{\mathbf{s}_{2}}\right)_{-}$over the channel in time, see also Figure 1 as

$$
\mathbf{h} *\left[\left(\begin{array}{c}
0 \\
C P 1 \\
\mathbf{s}_{1} \\
\mathbf{0}_{L} \\
\mathbf{0}_{N}
\end{array}\right)+\left(\begin{array}{c}
0 \\
\mathbf{0}_{L} \\
\mathbf{0}_{N} \\
C P 2 \\
\left(\overline{\left.\mathbf{s}_{2}\right)_{-}}\right.
\end{array}\right)\right]=\left(\begin{array}{c}
\mathbf{g}_{1} \\
\mathbf{y}_{1} \\
\mathbf{g}_{2}+\mathbf{g}_{3} \\
\mathbf{y}_{2} \\
\mathbf{g}_{4}
\end{array}\right) \in \mathbb{C}^{2 N+3 L}
$$

where $\left\{\mathbf{g}_{i}\right\}_{i=1}^{4} \subset \mathbb{C}^{L}$ are "garbage" vectors due to the cyclic prefix. Hence in the noiseless case we receive by (12)

$$
\mathbf{y}_{1}=\left(\begin{array}{c}
\mathbf{h} \\
\mathbf{o}_{N-L}
\end{array}\right) \otimes \mathbf{s}_{1} \quad \text { and } \quad \mathbf{y}_{2}=\left(\begin{array}{c}
\mathbf{h} \\
\mathbf{o}_{N-L}
\end{array}\right) \otimes\left(\overline{\mathbf{s}_{2}}\right)_{-} .
$$




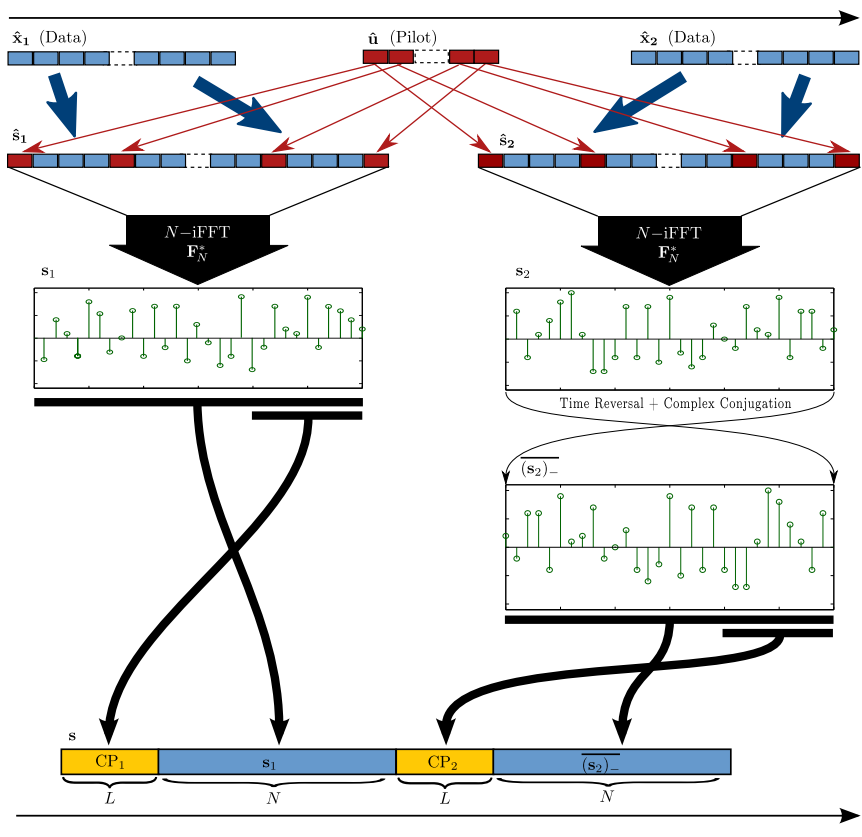

Figure 1. Scheme of the OFDM transmitter using phaseless pilots

By applying complex-conjugation and time-reversal on $\mathbf{y}_{2}$ and shifting afterwards circular by -1 , we can reverse the flipping on $\mathbf{s}_{2}$ and flip instead the channel:

$$
\mathbf{S}^{-1}\left(\overline{\mathbf{y}_{2}}\right)_{-}=\mathbf{S}^{-1}\left(\left(\begin{array}{c}
\overline{\mathbf{h}} \\
\mathbf{0}_{N-L}
\end{array}\right) \otimes\left(\mathbf{s}_{2}\right)_{-}\right)_{-}=\left(\begin{array}{c}
\mathbf{0}_{N-L} \\
\overline{\mathbf{h}_{-}}
\end{array}\right) \circledast \mathbf{S}_{2}
$$

If we take the DFT of the sum of $\mathbf{S y}_{1}$ and $\mathbf{S}^{-1}\left(\overline{\mathbf{y}_{2}}\right)_{-}$- we get:

$$
\begin{aligned}
\hat{\mathbf{y}} & =\left(\mathbf{F S y}_{1}+\mathbf{F S}^{-1}\left(\overline{\mathbf{y}_{2}}\right)_{-}\right)_{\mathcal{P}} \\
& =\sqrt{N}\left[\left(\mathbf{F}\left(\begin{array}{c}
0 \\
\mathbf{h} \\
\mathbf{0}_{N-L-1}
\end{array}\right)\right) \odot \hat{\mathbf{s}}_{1}+\left(\mathbf{F}\left(\begin{array}{c}
0 \\
\mathbf{0}_{N-L-1} \\
\overline{\mathbf{h}}-
\end{array}\right)\right) \odot \hat{\mathbf{s}}_{2}\right]_{\mathcal{P}} .
\end{aligned}
$$

Since it holds by construction of the symbols $\left(\hat{\mathbf{s}}_{1}\right)_{\mathcal{P}}=\left(\hat{\mathbf{s}}_{2}\right)_{\mathcal{P}}=$ $\hat{\mathbf{u}}$ we can factorize the pilots out by bilinearity of the convolution and get

$$
\hat{\mathbf{y}}=\sqrt{N}\left(\mathbf{F}\left(\begin{array}{c}
0 \\
\mathbf{h} \\
\mathbf{0}_{K} \\
\overline{\mathbf{h}}_{-}
\end{array}\right)\right)_{\mathcal{P}} \odot \hat{\mathbf{u}}=\sqrt{N} \mathbf{F}_{N} \mathcal{S}_{K}^{\circ}(\mathbf{h}) \odot \hat{\mathbf{u}},
$$

with $K=N-2 L-1$. By assumption $N$ is even and hence $K$ is always odd. Hence we obtained the Fourier transform of the conjugate-symmetrized form $\mathcal{S}_{K}^{\circ}(\mathbf{h})$ of the impulse response $\mathbf{h}$ in $N$ dimensions. Since the $P$ pilots are uniformly spaced in $[N]$ with distance $D$ we have $N=D P$. If we also assume $P$ to be even (we could also consider $P$ to be odd, but then we need another symmetrization) we only need $K^{\prime}=P-2 L-1$ odd zeros between $\mathbf{h}$ and $\overline{\mathbf{h}}_{-}$by applying, as in the classical case, the uniform circular sampling theorem (Nyquist Sampling)

$$
\left(\mathbf{F}_{N} \mathcal{S}_{K}^{\circ}(\mathbf{h})\right)_{\mathcal{P}}=\sqrt{\frac{P}{N}} \mathbf{F}_{P} \mathcal{S}_{K^{\prime}}^{\circ}(\mathbf{h})=\frac{1}{\sqrt{D}} \mathbf{F}_{P} \mathcal{S}_{K^{\prime}}^{\circ}(\mathbf{h})
$$

which is again the Fourier transform of a circular conjugatesymmetric vector, but now in only $P$ dimensions, since the Fourier measurements in (16) are actually an oversampling by the factor $D$. Hence, taking the square of the absolute-values in (16) gives our noise-free processed Fourier measurements

$$
\mathbf{z}=\frac{\operatorname{diag}\left(|\hat{\mathbf{u}}|^{-2}\right)}{P}|\hat{\mathbf{y}}|^{2}=\left|\mathbf{F}_{P} \mathcal{S}_{K^{\prime}}^{\circ}(\mathbf{h})\right|^{2}
$$

which constitute therefore a phase retrieval problem from quadratic measurements:

$\left(P_{\text {quad }}\right) \quad$ find $\mathbf{h} \in \mathbb{C}^{L}$ s.t. $\mathbf{z}=\left|\mathbf{F}_{P} \mathcal{S}_{K^{\prime}}^{\circ}(\mathbf{h})\right|^{2}$.

By [5], shown in [15], we can recover in the noise-free case $\mathbf{h}$ from the processed Fourier measurements $\mathbf{z}$ up to a global sign, i.e., the problem $\left(P_{\text {quad }}\right)$ has a unique solution up to global sign. This should be compared to "pure" Fourier measurements where recovery is up to several ambiguities, as already mentioned above (or see again [8]). The main idea here is to use the real-valued property of the symmetrized channel $\mathcal{S}_{K}^{\circ}(\mathbf{h})$. Note, that $\hat{\mathbf{y}}$ is not real valued, since the pilots $\hat{\mathbf{u}}$ are not real valued. Hence taking the absolute value eliminates the unknown pilot phases but keeps all available channel information at the receiver except for a global sign.

Before proceeding to reconstruction algorithms we shall elaborate more on symmetrization as a real-valued mapping. We will rewrite the symmetrization operator $\mathcal{S}_{K^{\prime}}^{\circ}$, which is a "non-complex-linear" map from $\mathbb{C}^{L}$ to $\mathbb{C}^{P}$ as a linear map $\boldsymbol{\Lambda}: \mathbb{R}^{2 L} \rightarrow \mathbb{C}^{P}$. Let be $\mathbf{g}=\left(\begin{array}{l}\mathbf{c} \\ \mathbf{d}\end{array}\right) \in \mathbb{R}^{2 L}$ where $\mathbf{c}=\operatorname{Re}(\mathbf{h}) \in \mathbb{R}^{L}$ and $\mathbf{d}=\operatorname{Im}(\mathbf{h}) \in \mathbb{R}^{L}$. Then:

$$
\mathbf{x}=\mathcal{S}_{K^{\prime}}^{\circ}(\mathbf{h})=\left(\begin{array}{c}
0 \\
\mathbf{c}+i \mathbf{d} \\
\mathbf{0}_{K^{\prime}} \\
\mathbf{c}_{-}-i \mathbf{d}_{-}
\end{array}\right)=\left(\begin{array}{cc}
\mathbf{0}_{1 \times L} & \mathbf{0}_{1 \times L} \\
\mathbb{1}_{L} & i \mathbb{1}_{L} \\
\mathbf{K}_{K^{\prime} \times L} & \mathbf{0}_{K^{\prime}} \\
\mathbf{R}_{L} & -i \mathbf{R}_{L}
\end{array}\right)\left(\begin{array}{l}
\mathbf{c} \\
\mathbf{d}
\end{array}\right)=: \mathbf{\Lambda} \mathbf{g} .
$$

where $\mathbf{R}_{L}$ denotes the matrix for time-reversal, see (1). This gives the following equivalent of $(5)$ for any $\mathbf{g}_{1}, \mathbf{g}_{2} \in \mathbb{R}^{2 L}$

$$
\alpha\left\|\mathbf{g}_{1}-\mathbf{g}_{2}\right\|_{2}\left\|\mathbf{g}_{1}+\mathbf{g}_{2}\right\|_{2} \leq\left\|\left|\widehat{\mathbf{g}_{1}}\right|^{2}-\left|\widehat{\mathbf{\Lambda g}_{2}}\right|^{2}\right\|_{2} .
$$

We can even get rid of the squares in our absolute values by using the binomial formula by setting $\hat{\mathbf{x}}_{i}=\overline{\mathbf{\Lambda g}}_{i}$ for $i=1,2$.

$$
\begin{aligned}
\|\left|\hat{\mathbf{x}}_{1}\right|^{2} & -\left|\hat{\mathbf{x}}_{2}\right|^{2}\left\|_{2}=\right\|\left(\left|\hat{\mathbf{x}}_{1}\right|-\left|\hat{\mathbf{x}}_{2}\right|\right) \odot\left(\left|\hat{\mathbf{x}}_{1}\right|+\left|\hat{\mathbf{x}}_{2}\right|\right) \|_{2} \\
& =\left\|\left(\left|\hat{\mathbf{x}}_{1}\right|-\left|\hat{\mathbf{x}}_{2}\right|\right)^{2} \odot\left(\left|\hat{\mathbf{x}}_{1}\right|+\left|\hat{\mathbf{x}}_{2}\right|\right)^{2}\right\|_{1}^{\frac{1}{2}} \\
& \leq\left(\left\|\left(\left|\hat{\mathbf{x}}_{1}\right|-\left|\hat{\mathbf{x}}_{2}\right|\right)^{2}\right\|_{2} \cdot\left\|\left(\left|\hat{\mathbf{x}}_{1}\right|+\left|\hat{\mathbf{x}}_{2}\right|\right)^{2}\right\|_{2}\right)^{\frac{1}{2}} \\
& =\left\|\left|\hat{\mathbf{x}}_{1}\right|-\left|\hat{\mathbf{x}}_{2}\right|\right\|_{4} \cdot\left\|\left|\hat{\mathbf{x}}_{1}\right|+\left|\hat{\mathbf{x}}_{2}\right|\right\|_{4} \\
& \leq\left\|\left|\hat{\mathbf{x}}_{1}\right|-\left|\hat{\mathbf{x}}_{2}\right|\right\|_{4}\left(\left\|\left|\hat{\mathbf{x}}_{1}\right|^{2}\right\|_{2}^{\frac{1}{2}}+\left\|\left|\hat{\mathbf{x}}_{2}\right|^{2}\right\|_{2}^{\frac{1}{2}}\right) .
\end{aligned}
$$

Due to the conjugate-symmetry and the zero-padding in $\mathbf{x}_{i}$ we can upper bound (using Parseval-identity, Young and CauchySchwartz inequality) to get: $\left\|\left|\hat{\mathbf{x}}_{i}\right|^{2}\right\|_{2}=\left\|\boldsymbol{\Lambda} \mathbf{g}_{i} \otimes \Lambda \mathbf{g}_{i}\right\|_{2}=$ $\left\|\boldsymbol{\Lambda} \mathbf{g}_{i} * \boldsymbol{\Lambda} \mathbf{g}_{i}\right\|_{2} \leq \sqrt{2 L}\left\|\mathbf{g}_{i}\right\|_{2}^{2}=\sqrt{2 L}\left\|\mathbf{h}_{i}\right\|_{2}^{2}$. Assuming an upper bound $\left\|\mathbf{h}_{i}\right\|_{2} \leq \eta$ gives therefore the weaker stability constant:

$$
\alpha \cdot \frac{\left\|\mathbf{g}_{1}-\mathbf{g}_{2}\right\|\left\|\mathbf{g}_{1}+\mathbf{g}_{2}\right\|}{2(2 L)^{\frac{1}{4}} \eta} \leq\left\|\left|\widehat{\mathbf{\Lambda} \mathbf{g}_{1}}\right|-\left|\widehat{\Lambda \mathbf{g}_{2}}\right|\right\|_{4} .
$$

Thus, increasing the power $\eta$ decreases the stability of the map $|\widehat{\Lambda g}|$. We will see in the next paragraph, that in the noisy case that this has impact on $\left(P_{\text {quad }}\right)$. However, in the noiseless case the problem based on (non-squared) absolute-values in 18

$$
\left(P_{\text {abs }}\right) \quad \text { find } \mathbf{g} \in \mathbb{R}^{2 L} \text { s.t. } \mathbf{z}=\left|\mathbf{F}_{P} \boldsymbol{\Lambda} \mathbf{g}\right| \text {. }
$$


is equivalent to $\left(P_{\text {quad }}\right)$. Moreover, $\left(P_{\text {abs }}\right)$ constitute a generalized phase retrieval problem for real-valued vectors.

$g$ ) The Noisy Versions of $\left(P_{\text {quad }}\right)$ and $\left(P_{a b s}\right)$ : Applying additive noise to the linear Fourier measurements prior to taking magnitudes comes therefore with a noise model that is usually not investigated by the phase retrieval community. Let us consider additive complex-valued noise $\mathbf{n}_{1}, \mathbf{n}_{2} \in \mathbb{C}^{N}$ in (7) affecting channel estimation and data demodulation. Let us set $\boldsymbol{\alpha}=|\hat{\mathbf{u}}|^{-1} / \sqrt{P}$ and compute for the absolute-valued measurements

$$
\begin{aligned}
\mathbf{b} & =\boldsymbol{\alpha} \odot\left|\mathbf{F}\left[\mathbf{S}\left(\mathbf{y}_{1}+\mathbf{n}_{1}\right)+\mathbf{S}^{-1}\left(\overline{\mathbf{y}_{2}+\mathbf{n}_{2}}\right)_{-}\right]\right|_{\mathcal{P}} \\
& =: \boldsymbol{\alpha} \odot|\underbrace{\left(\mathbf{F}\left[\mathbf{S}\left(\mathbf{y}_{1}\right)+\mathbf{S}^{-1} \overline{\mathbf{y}_{2}}\right]\right)_{\mathcal{P}}}_{=\hat{\mathbf{y}}}+\hat{\mathbf{n}}| .
\end{aligned}
$$

Absolute-squared measurements contain therefore a cross term $\boldsymbol{\alpha} \odot(\hat{\mathbf{y}} \odot \overline{\hat{\mathbf{n}}}+\overline{\hat{\mathbf{y}}} \cdot \hat{\mathbf{n}})$ between noise and the symmetrized channel. The residual is therefore $\mathbf{b}^{2}-\boldsymbol{\alpha}^{2} \odot|\hat{\mathbf{y}}|^{2}=\boldsymbol{\alpha}^{2} \odot(\hat{\mathbf{y}} \odot \overline{\hat{\mathbf{n}}}+$ $\left.\overline{\hat{\mathbf{y}}} \odot \hat{\mathbf{n}}+|\hat{\mathbf{n}}|^{2}\right)$ and increasing SNR increases the residual as well. Therefore, careful adjustment of an effective (practically reasonable) bound $\epsilon_{\text {eff }}$ on the expected residuals depending apriori-knowledge or on the statistic of system parameters is mandatory for the quadratic problem:

$$
\left(P_{\text {quad, } \epsilon_{\text {eff }}}\right) \quad \text { find } \mathbf{g} \in \mathbb{R}^{2 L} \text { s.t. }\left\|\mathbf{b}^{2}-\left|\mathbf{F}_{P} \boldsymbol{\Lambda} \mathbf{g}\right|^{2}\right\|_{2} \leq \epsilon_{\text {eff }}
$$

For the (non-squared) absolute-valued problem $\left(P_{\text {abs }}\right)$ we compute the residual between $\mathbf{b}$ in 26 and the noise-free version $\mathbf{z}$ in 18 using reverse triangle inequality:

$$
\|\mathbf{b}-\mathbf{z}\|_{2}=\|\boldsymbol{\alpha} \odot(|\hat{\mathbf{y}}+\hat{\mathbf{n}}|-|\hat{\mathbf{y}}|)\|_{2} \leq\|\boldsymbol{\alpha} \odot \hat{\mathbf{n}}\|_{2} .
$$

From our stability result and the fact that $f(\mathbf{g})=|\widehat{\Lambda} \mathbf{g}|=\mathbf{z}$ is convex we have the following convex optimization problem for the noisy phase retrieval problem for absolute Fourier measurements of symmetrized signals:

$\left(P_{\text {abs }, \epsilon}\right) \quad$ find $\mathbf{g} \in \mathbb{R}^{2 L}$ s.t. $\left\|\mathbf{b}-\left|\mathbf{F}_{P} \mathbf{\Lambda} \mathbf{g}\right|\right\|_{2} \leq \epsilon$

where we simplified by $\left|\alpha_{k}\right|=1$ and $\|\hat{\mathbf{n}}\|_{2} \leq \epsilon$. Obviously, this problem is still ill-posed. We will postpone the investigation of $\left(P_{\text {abs }, \epsilon}\right)$ to a companion paper. The problem can not be solved directly using an linear estimator as compared to $\left(P_{\text {quad, }, \epsilon_{\mathrm{eff}}}\right)$ (without further assumptions).

\section{AlgORITHMS}

For the quadratic problem $\left(P_{\mathrm{abs}, \epsilon}\right)$ we propose here two different reconstruction strategies. First, we tackle the problem in a direct way by lifting the quadratic problem to a linear low-rank matrix recovery problem. Its convex relaxation can be efficiently solved by a semidefinite program. This approach follows the same lifting ideas recently used in phase retrieval by [4] but with specific (not Gaussian, not generic and even not Fourier), structured measurements. Although this is motivated by the noise-robustness of such programs its practical implementation is at this time not yet feasible for the desired application.

The second strategy is a two-stage approach, where we first solve the linear problem of estimating the auto-convolution of the symmetrized channel and afterwards performing an iterative deconvolution algorithm to extract the channel. Nevertheless, the iterative "de-autoconvolution" has also its stability issue and is in general difficult to address, see e.g. [16].

\section{A. Recovery via a Semidefinite Program}

For any $\mathbf{x} \in \mathbb{C}^{P}$ we can write $\left|\mathbf{F}_{P} \mathbf{x}\right|^{2}$ as a linear map of a rank-1 matrix $\mathbf{x x}^{*}$, which is known as the (phase) lifting technique [4],[21]. For each $k \in[P]$ we have:

$$
\left(\left|\mathbf{F}_{P} \mathbf{x}\right|^{2}\right)_{k}=\operatorname{tr}\left(\check{\mathbf{e}}_{k} \check{\mathbf{e}}_{k}^{*} \mathbf{x} \mathbf{x}^{*}\right)
$$

and overall this maps complex-valued positive matrices to positive real-valued vectors. Incorporating the conjugatesymmetric structure $\mathbf{x}=\Lambda \mathbf{g}$ in 30 yields for $k \in[P]$

$$
z_{k}=\left|\mathbf{F}_{P} \boldsymbol{\Lambda} \mathbf{g}\right|_{k}^{2}=\operatorname{tr}\left(\left(\boldsymbol{\Lambda}^{*} \check{\mathbf{e}}_{k} \check{\mathbf{e}}_{k}^{*} \boldsymbol{\Lambda}\right) \mathbf{g g}^{T}\right)=: \mathcal{A}\left(\mathbf{g g}^{T}\right)_{k} .
$$

where by the conjugate-symmetry property (2) this defines indeed a real-valued linear map $\mathcal{A}: \mathbb{R}^{2 L \times 2 L} \rightarrow \mathbb{R}^{P}$. This linear map can also be seen as the square of the superposition of an oversampled discrete cosine transform on the real part and an oversampled discrete sine transform on the imaginary part of h. Similar approaches are used also in [22-24]. From (5) we know that only one positive rank-1 matrix $\mathbf{G}=\mathbf{g g}^{T} \in \mathbb{R}^{2 L \times 2 L}$ exists giving noise-free measurements $\mathbf{z}$ in $(18)$. The minimal amount of any real-valued linear measurements to guarantee unique reconstruction up to global sign is $2(2 L)-1=4 L-1$, which can be achieved by generic linear measurements [5]. In our case we found $P \geq 4 L+2$ deterministic real-valued linear measurements to guarantee recovery up to global sign. In fact, the leading zero for the channel symmetrization could even be omitted by assuming $h_{0}$ to be real, which would give unique recovery of $2 L-1$ real-valued unknown from only $4 L-2$ magnitudes of linear measurements. Hence we end up with the following rank minimizing optimization

$$
\mathbf{G}=\underset{\tilde{\mathbf{G}} \in \mathbb{R}^{2 L \times 2 L}, \tilde{\mathbf{G}} \geq \mathbf{0}}{\operatorname{argmin}} \operatorname{rk}(\tilde{\mathbf{G}}) \quad \text { s.t. } \quad \mathbf{z}=\mathcal{A}(\tilde{\mathbf{G}})
$$

which is equivalent to $\left(P_{\text {quad }}\right)$. The real vector $\mathbf{g}$ is obtained from the SVD of $\mathbf{G}$ and hence $\mathbf{h}=\left(g_{0}+i g_{L}, \ldots, g_{L-1}+\right.$ $\left.i g_{2 L-1}\right)^{T}$ up to global sign. To determine the global sign it is sufficient to know one of the phases of the pilot tones or one can even reconstruct this from the data under certain assumptions. The following convex relaxation of 32 is wellestablished in phase retrieval [25],[26], [21]:

$$
\mathbf{G}=\underset{\tilde{\mathbf{G}} \in \mathbb{R}^{2 L \times 2 L}, \tilde{\mathbf{G}} \geq \mathbf{0}}{\operatorname{argmin}} \operatorname{tr}(\tilde{\mathbf{G}}) \quad \text { s.t. } \quad \mathbf{z}=\mathcal{A}(\tilde{\mathbf{G}})
$$

In fact, one still has to show that all other semidefinitepositive matrices which are feasible solutions $\left\{\tilde{\mathbf{G}} \in \mathbb{R}^{2 L \times 2 L} \mid \mathcal{A}(\tilde{\mathbf{G}})=\mathbf{z}\right\}$ yielding strictly larger traces. To derive such results further assumptions on the signals are needed [27]. At time of writing it is not clear to what extent the conjugate-symmetric property is sufficient here. In our simulation we observed that there might exists other matrices of higher rank yielding smaller traces than $\mathbf{g g}^{T}$, although for highly sparse channels $\left(P_{*}\right)$ seems to yield satisfying results. 
In this case we can promote sparse matrices in $\left(P_{*}\right)$ by the convex function $\lambda\|\mathbf{G}\|_{1}$ for some parameter $\lambda>0$ :

$$
\left(P_{*, \epsilon_{\mathrm{eff}}}\right) \min _{\substack{\tilde{\mathbf{G}} \in \mathbb{R}^{2 L \times 2 L} \\ \tilde{\mathbf{G}} \geq \mathbf{0}}} \operatorname{tr}(\tilde{\mathbf{G}})+\lambda\|\tilde{\mathbf{G}}\|_{1} \text { s.t. }\|\mathbf{z}-\mathcal{A}(\tilde{\mathbf{G}})\|_{2} \leq \epsilon_{\mathrm{eff}}
$$

where the discussion on the effective bound $\epsilon_{\text {eff }}$ above is relevant again, i.e., $\epsilon_{\text {eff }}$ has to be manually adjusted with respect to prior-knowledge on $\mathbf{h}$ or to some statistical parameters. The problem above is a semidefinite program and it can be solved using, for example, Sedumi. On the other, from practical perspectives such algorithms are far from being usable in the context of channel estimation due to complexity reasons. Furthermore, at time of writing, this does not provide a sufficiently robust channel estimation for practical relevant SNR values which is caused by the noise cross-term issues described above. Therefore we propose an iterativedeconvolution algorithm, which indeed produces similar BER rates for the channel estimation as in the classical cases with known pilot phases, see Figure 2

\section{B. Two-Stage Iterative Recovery}

The absolute-square Fourier measurements of $\mathcal{S}_{K^{\prime}}^{\circ}(\mathbf{h})$ can also be seen as the DFT of the circular auto-correlation of $\mathcal{S}_{K^{\prime}}^{\circ}(\mathbf{h})$. Due to the conjugate-symmetry this equals the circular auto-convolution with

$$
\mathcal{S}_{K^{\prime}}^{\circ}(\mathbf{h}) \circledast \mathcal{S}_{K^{\prime}}^{\circ}(\mathbf{h})=\mathbf{S}^{-2 L}\left(\underset{\mathbf{0}_{P-4 L-1}}{\mathbf{a}}\right)
$$

with $\mathbf{a}=\mathcal{S}_{0}^{\circ}(\mathbf{h}) * \mathcal{S}_{0}^{\circ}(\mathbf{h}) \in \mathbb{C}^{4 L+1}$ since $P \geq 4 L+1$ and $\mathbf{h} \in \mathbb{C}^{L}$, see also [1] where we also discuss the case of non-uniform pilots, due to dimensions mismatches. Due to the conjugatesymmetry of a it is also sufficient to estimate only the onesided autoconvolution, i.e., optimize in the first stage over vectors in $\mathbb{C}^{M}$ where $M=2 L+1$ instead of $4 L+1$.

h) Least-Squares Estimation of the Auto-convolution: Hence, the first stage is a least-square (LS) estimation of the auto-convolution a from the square of the noisy measurements b in 26) given by

$$
\min _{\tilde{\mathbf{a}} \in \mathbb{C}^{M}}\left\|\mathbf{b}^{2}-\mathbf{A}^{(\mathrm{p})} \tilde{\mathbf{a}}\right\|
$$

with the linear map $\mathbf{A}^{(\mathrm{p})}=\mathbf{F S}^{-2 L} \mathbf{Q} \in \mathbb{C}^{P \times M}$ where $\mathbf{Q x}$ zeropads ã to $P$ dimension. Similar to 10 we can consider here the family:

$$
\left(\mathbf{A}^{(\mathrm{p})^{*}} \mathbf{A}^{(\mathrm{p})}+\tau\right)^{-1} \mathbf{A}^{(\mathrm{p})^{*}} \mathbf{b}^{2}
$$

of estimates for depending on a choosen parameter $\tau$.

i) Estimation of the Auto-convolution under Sparsity Prior: If the channel impulse response is known to be $S$ sparse its symmetrized version is at most $2 S$-sparse and hence its auto-convolution is at most $\left(2 S^{2}+S\right)$-sparse.

$$
\min _{\tilde{\mathbf{a}} \in \mathbb{C}^{M}}\|\tilde{\mathbf{a}}\|_{0} \quad \text { s.t. } \quad\left\|\mathbf{b}^{2}-\mathbf{A}^{(\mathrm{p})} \tilde{\mathbf{a}}\right\|_{2} \leq \epsilon_{\mathrm{eff}} .
$$

If $\mathbf{A}^{\text {(p) }}$ acts almost isometric on the desired ã's the problem above can be relaxed (37) to the (convex) basis pursuit denoising problem

$$
\min _{\tilde{\mathbf{a}} \in \mathbb{C}^{4 L+1}}\|\tilde{\mathbf{a}}\|_{1} \quad \text { s.t. } \quad\left\|\mathbf{b}^{2}-\mathbf{A}^{(\mathrm{p})} \tilde{\mathbf{a}}\right\|_{2} \leq \epsilon_{\text {eff }}
$$

But, due to the increased sparsity of the auto-convolution with symmetrization the gains are limited here for practical settings. As already discussed the overall compressibility due to a sparse channel can not fully taken into account here since the original problem is an sparse rank-one problem. Furthermore, randomizing pilot position in a fashion known to the receiver increases again the amount of prior-knowledge at the receiver which we want to avoid.

j) Iterative De-Autoconvolution, Noiseless Case: For a first exposition we start with the noiseless case and the ideas was inspired by the work of [22] and [23]. A similar approach has been used in [24]. However, the algorithms proposed in these works are not noise robust. An overall stable "de-autoconvolution" is indeed an unsolved problem [16]. Nevertheless, we present an approach of a manually adopted iterative Tikhonov regularization. The values $h_{0} \ldots h_{L-1}$ are determined up to global sign by $a_{0} \ldots a_{L-1}$ due to:

$$
a_{k}=\sum_{l=0}^{k} h_{l} h_{k-l}
$$

To see this, let us assume that $a_{0}$ is non-zero and real (multiplying a by a global phase). Then it follows from 39 that $a_{0}=h_{0}^{2}$, i.e., $h_{0}= \pm \sqrt{a_{0}}$. Given the values $h_{0} \ldots h_{k-1}$ one can can solve for $h_{k}$ using again 39.

$$
h_{k}=\frac{a_{k}-\sum_{l=1}^{k-1} h_{l} h_{k-l}}{2 h_{0}} .
$$

At this point it is clear that dividing by $h_{0}$ could make this algorithm quite unstable in the noisy setting. For practical purposes it is therefore important to estimate $h_{0}$ with high accuracy and to order operations by magnitudes (which we will not consider here).

k) Noisy Case, Thresholding and Regularization: Let $\mathbf{X}=\mathbf{h h}^{T}$ be the rank-one $L \times L$ matrix associated to the vector $\mathbf{h}$. Since $\mathbf{h} \in \mathbb{C}^{L}$ is complex the matrix $\mathbf{X}=\left(X_{i j}\right) \in \mathbb{C}^{L \times L}$ is not positive semi-definite. Let $X_{i j}=\left\langle\mathbf{e}_{i}, \mathbf{X} \mathbf{e}_{j}\right\rangle=\operatorname{tr}\left(\mathbf{X} \mathbf{e}_{i} \mathbf{e}_{j}^{T}\right)$ be the elements of $\mathbf{X}$ where $\mathbf{e}_{i}$ are the real standard basis vectors. We can write therefore:

$$
a_{k} \stackrel{39]}{-} \sum_{l=0}^{k} X_{l, k-l}=\operatorname{tr}\left(\mathbf{X} \sum_{l} \mathbf{e}_{k-l} \mathbf{e}_{l}^{T}\right)=: \operatorname{tr}\left(\mathbf{E}_{k} \mathbf{X}\right)
$$

where the matrix $\mathbf{E}_{k}$ shifts coordinates $1 \ldots k$ (in the standard basis) of a vector by $k$ positions. Consistent with our first illustration of the noiseless case above, this means that $\operatorname{tr}\left(\mathbf{E}_{k} \mathbf{X}\right)$ depends only on $h_{0} \ldots h_{k}$. Although this is advantageous from complexity point of view this is also the reason for error accumulation. On the other hand the equation above allows for post-processing the values $h_{0} \ldots h_{k}$ by a least-squares approach with regularization in the sense of Tikhonov. This regularization has already been investigated and proposed for general autoconvolution problems, see [16]. More precisely, let $\mathbf{a}^{\#}$ be the estimate of the autoconvolution obtained from stage one in a noisy setup. In applications, the channels to be estimated are often sparse or compressible, i.e. for example $\mathbf{h}$ is $S$-sparse. Thus it is reasonable to sparsify 
noisy measurements of its autoconvolution by a thresholding procedure:

$$
\mathbf{a}^{\text {thr }}=\mathbf{a}^{\#} \cdot \mathbf{1}\left(k>0:\left|a_{k}^{\#}\right| \geq \lambda\right) .
$$

In particular, $k=0$ will be ignored here since it will be used to determine $h_{0}$ (although there would be alternatives). Hard thresholding in the order of a fraction of the universal threshold according to [28] (for Gaussian noise) would suggest a thresholding level of

$$
\lambda \approx 0.1 \sqrt{\sigma_{\mathrm{eff}}^{2}(4 L+1)}
$$

In our experiments we observed that this already gives a substantial improvement. However, in real applications this ratio has obviously to be tuned to the environment and to the statistics of all random contributions.

The least-squares solution $\mathbf{h}$ for $\mathbf{a}^{\text {thr }}$, often used due to its simplicity, would minimize the $\ell_{2}$-norm of the following residual:

$$
\mathbf{a}^{\text {thr }}-\sum_{k} \operatorname{tr}\left(\mathbf{E}_{k} \mathbf{h} \mathbf{h}^{T}\right) \mathbf{e}_{k} .
$$

Minimizing such an objective (locally, since it is not convex in the vector $\mathbf{h}$ ) results in considerable strong fluctuations of $\|\mathbf{h}\|_{2}$ since the auto-convolution problem itself is ill-posed. A common approach here is the following regularization:

$$
\min _{\mathbf{h}}\left\|\mathbf{a}^{\mathrm{thr}}-\sum_{k} \operatorname{tr}\left(\mathbf{E}_{k} \mathbf{h} \mathbf{h}^{T}\right) \mathbf{e}_{k}\right\|_{2}^{2}+\alpha\|\boldsymbol{\Omega} \mathbf{h}\|_{2}^{2} .
$$

Since the objective is non-convex it can with moderate complexity only be minimized locally (which itself can be implemented efficiently again), i.e., a reasonable initial point is necessary. Furthermore, we use $\boldsymbol{\Omega}=\operatorname{diag}\left(e^{(L-1) \omega}, \ldots, e^{0 \omega}\right)$ with $\omega=4$ to perform a reweighting. This causes a decreasing influence of "past" values to the current iteration $k$. Together with the observation that $\operatorname{tr}\left(\mathbf{E}_{k} \mathbf{h} \mathbf{h}^{T}\right)$ depends only on the values $h_{0} \ldots h_{k}$ we propose the following successive approximation algorithm (see the algorithm below). First, assume that values $h_{0} \ldots h_{k-1}$ are given. Then the value of $h_{k}$ is obtained according to (39). We use then this $k+1$-dimensional vector containing $h_{0} \ldots h_{k}$ as the initial point for locally minimizing (45) to get an update.

Let us call the updated values (the argmin in (45)) again with $h_{0} \ldots h_{k}$. As an intermediate operation we apply a pruning step, i.e., we keep only the $k^{\text {prun }}$ values having largest magnitude. Again this parameter has to be setup depending on the conditions. In our tests we assume that the channel $S_{-}$ sparse (or we are interested only in the $S$ values with largest magnitude) and we have used $k^{\text {prun }}=3 S / 2$.

The procedure above is iterated up to $k=L$ and finally $\mathbf{h}$ is again pruned to the $S$ entries with largest magnitude. Using this overall iterative algorithm we observed that it possible stabilize the de-auto-convolution.

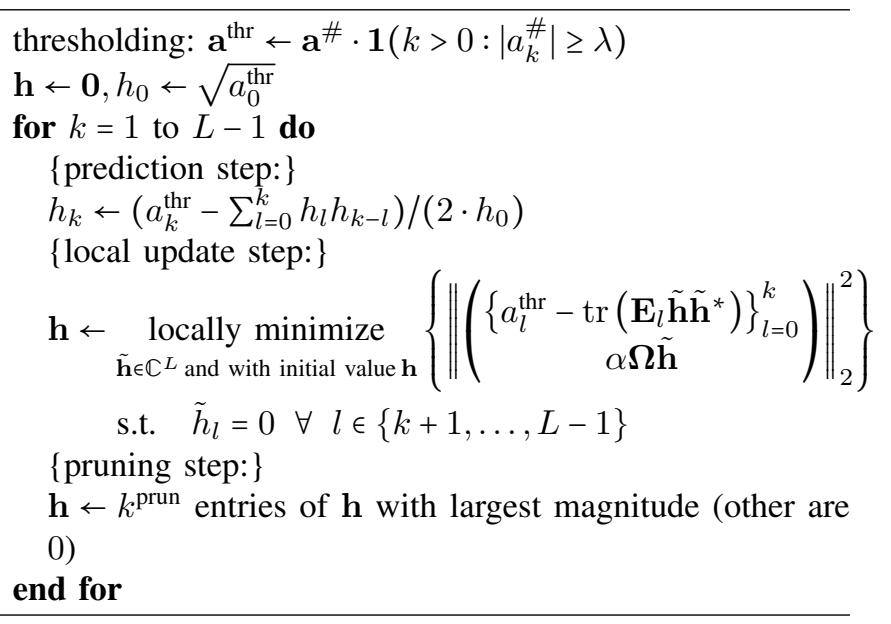

\section{Performance Evaluation}

We have evaluated the final performance in terms of (uncoded) bit error rates in a physical layer OFDM simulation. We observed that under moderate system conditions similar performance can be obtained for phaseless estimation as compared to conventional pilot-aided channel estimation, see Fig. 2. On the other hand, we also note that at the time of

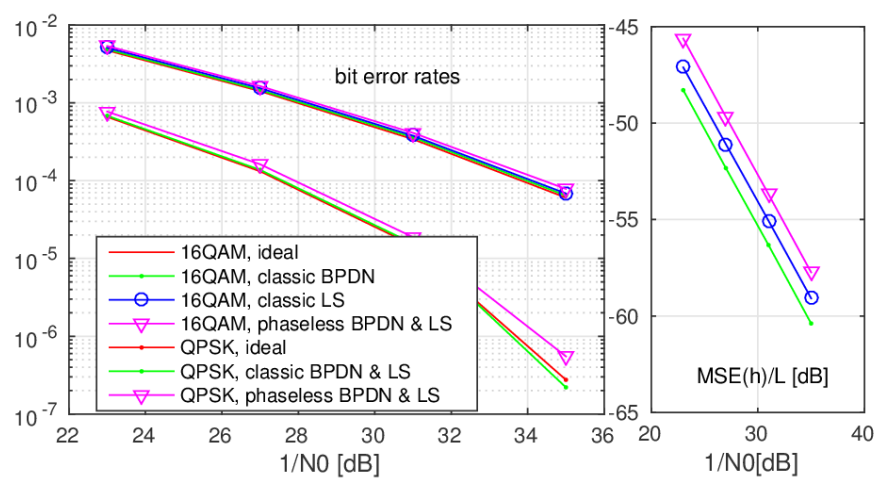

Figure 2. Bit error rate and mean squared error (MSE) per dimension for QPSK and 16QAM modulation. The parameters are $N=2048, L=20$, $P=256, S=3$ and $\alpha=0.01$. Classical least-squares (LS) and BPDN channel estimation is plotted in blue and green. Red denotes ideal channel knowledge. The cyan curve is the phaseless scheme where the autoconvolution is LS- or BPDN-estimated (no difference). The x-axis is inverse power $N_{0}$ $[\mathrm{dB}]$, i.e., the variance of i.i.d. complex normal distributed noise $\hat{\mathbf{n}}$.

writing the iterative algorithm for phaseless estimation still require careful adjustment of several parameters, as discussed above.

\section{CONClusion}

We have presented a novel semi-blind channel estimation method in using phaseless pilots for OFDM. With our approach the pilot phases are available again for user-dependent information. We have discussed the relation and the differences to phase retrieval using Fourier measurement and proposed a lifting method for our setup. On the numerical side we have established a two-stage algorithm which first estimates the auto-convolution and then proceeds by recovering iteratively 
the channel impulse response in a regularized fashion. Our results show that under moderate system assumptions it is possible to stabilize the "de-autoconvolution" and similar performance in BER can be obtained as compared to classical channel estimation using pilot phases.

Acknowledgments. We would like to thank Holger Boche for helpful discussions. This work was partially supported by the DFG grant JU 2795/2 and WA 3390/1.

[1] P. Walk, H. Becker, and P. Jung, "Phaseless pilots for ofdm", in International Symposium on Wireless Communication Systems, ISWCS15, Brussels, 2015.

[2] R. W. Gerchberg and W. O. Saxton, "A practical algorithm for the determination of phase from image and diffraction plane pictures", Optik, vol. 35, 237-246, 1972.

[3] J. R. Fienup, "Reconstruction of an object from the modulus of its fourier transform", Optics Letters, vol. 3, no. 1, pp. 27-29, 1978.

[4] E. J. Candès, T. Strohmer, and V. Voroninski, "Phaselift: exact and stable signal recovery from magnitude measurements via convex programming", Communications on Pure and Applied Mathematics, vol. 66, pp. 12411274, 2012.

[5] R. Balan, P. Casazza, and D. Edidin, "On signal reconstruction without phase", Applied and Computational Harmonic Analysis, vol. 20, pp. 345-356, 2006.

[6] R. Balan, B. G. Bodmann, P. Casazza, and D. Edidin, "Fast algorithms for signal reconstruction without phase", in Proc. SPIE 6701, 2007.

[7] D. Mondragon and V. Voroninski, "Determination of all pure quantum states from a minimal number of observables", arXiv:1306.1214, 2013.

[8] R. Beinert and G. Plonka, "Ambiguities in onedimensional discrete phase retrieval from fourier magnitudes", Journal of Fourier Analysis and Applications, 2015.

[9] Y. Wang, "Minimal frames for phase retrieval", in Workshop of phaseless recovery, 2013.

[10] Y. Lu and M. Vetterli, "Sparse spectral factorization: unicity and reconstruction algorithms", in Proc. IEEE International Conference on Acoustics, Speech and Signal Processing, Prague, 2011.

[11] Y. Eldar and S. Mendelson, "Phase retrieval: stability and recovery guarantees", Applied and Computational Harmonic Analysis, vol. 36, no. 3, pp. 473-494, 2014.

[12] M. Ehler, M. Fornasier, and J. Siegl, "Quasi-linear compressed sensing", SIAM: Multiscale Modeling and Simulation, vol. submitted, 2013.

[13] Y. Wang and Z. Xu, "Phase retrieval for sparse signals", arxiv, 2013.

[14] K. Jaganathan, S. Oymak, and B. Hassibi, "Sparse phase retrieval: uniqueness guarantees and recovery algorithms", Arxiv.org, 2013.
[15] P. Walk and P. Jung, "Stable recovery from the magnitude of symmetrized fourier measurements", in IEEE International Conference on Acoustics, Speech and Signal Processing (ICASSP), 2014, pp. 1813 -1816. DOI: 10.1109/ICASSP.2014.6853911

[16] R. Gorenflo and B. Hofmann, "On autoconvolution and regularizaition", Inverse Problems, vol. 70, pp. 353373, 1994.

[17] P. Walk, P. Jung, and G. E. Pfander, "On the stability of sparse convolutions", Applied and Computational Harmonic Analysis, 2015. DOI: doi:10.1016/j.acha.2 015.08.002, eprint: arXiv:1409.6874

[18] P. Jung and P. Walk, "Compressed sensing and its applications", in, H. Boche, A. R. Calderbank, G. Kutyniok., and J. Vybiral, Eds., ser. Applied and Numerical Harmonic Analysis. Springer, 2014, ch. Sparse Model Uncertainties in Compressed Sensing with Application to Convolutions and Sporadic Communication, pp. 283313.

[19] E. J. Candès, "The restricted isometry property and its implications for compressed sensing", Compte Rendus de l'Academie des Sciences, vol. 346, pp. 589-592, 2008.

[20] W. Bajwa, J. Haupt, A. Sayeed, and R. Nowak, "Compressed channel sensing: A new approach to estimating sparse multipath channels", Proceedings of the IEEE, vol. 98, no. 6, pp. 1058-1076, 2010.

[21] E. J. Candès, Y. Eldar, T. Strohmer, and V. Voroninski, "Phase retrieval via matrix completion", SIAM Journal on Imaging Sciences, vol. 6, no. 1, pp. 199-225, 2013.

[22] M. Fickus, D. G. Mixon, A. A. Nelson, and Y. Wang, "Phase retrieval from very few measurements", Linear Algebra and its Applications, vol. 449, 2014.

[23] B. G. Bodmann and N. Hammen, "Stable phase retrieval with low-redundancy frames", Advances in Computational Mathematics, vol. 41, no. 2, pp. 317-331, 2015.

[24] F. Philipp, "Phase retrieval from 4n-4 measurements", Arxiv, 2014.

[25] T. Cai and A. Zhang, "Sharp rip bound for sparse signal and low rank matrix recovery", Applied and Computational Harmonic Analysis, vol. 35, pp. 74-93, 2013.

[26] B. Recht, M. Fazel, and P. A. Parrilo, "Guaranteed minimum-rank solutions of linear matrix equations via nuclear norm minimization", SIAM Journal on Applied Mathematics, vol. 52, pp. 471-501, 2010.

[27] K. Jaganathan, Y. Eldar, and B. Hassibi, "Phase retrieval: an overview of recent developments", in arxiv, 2015. eprint: arXiv:1510.07713.

[28] D. L. Donoho and J. M. Johnston, "Ideal spatial adaptation by wavelet shrinkage", Biometrika, vol. 81, no. 3, pp. 425-455, 1994. 